\title{
A Review on Methods for Assessing Risk Factors of the Upper Limb Disorders among Cashiers in Grocery Retail Industries
}

\author{
Muhammad Fareez Ahmad Zuhaidi and Mohd Nasrull Abdol Rahman* \\ Department of Manufacturing and Industrial Engineering, Faculty of Mechanical Engineering and \\ Manufacturing, Universiti Tun Hussein Onn Malaysia (UTHM), 86400 Batu Pahat, Johor, Malaysia
}

\begin{abstract}
This paper provides an overview of the various methods that have been developed for the assessment of risk factors for upper limb disorders among cashiers in grocery retail industries. This paper is essential as upper limb disorders have been known as one of the prime cause of work-related disability in various countries. The methods used for the assessment were the Disabilities of the Arm, Shoulder and Hand (DASH) questionnaire, the Assessment of Repetitive Tasks (ART), the Job Strain Index (JSI) and the Occupational Repetitive Actions (OCRA) Checklist. DASH questionnaire is a structured interview in determining the prevalence of the upper limb disorders, while ART, the JSI and OCRA Checklist act as a direct observation in examining the involvement of risk factors. Basically, these tools dealt with the measurements of symptoms, posture observation, and workplace risk assessment, as they are the most relevant tools for assessing the risk factors faced by the workers in grocery industries. The use of these tools assessed the risk factors and consequently reduces the risk of injuries among the workers.
\end{abstract}

\section{Introduction}

Cashiers have been declared as one of top ten professions having the risk of developing musculoskeletal disorders (MSDs) concerning the upper limb [1]. Most grocery store managers and employees have carried out all sorts of efforts in reducing occupational injuries and illnesses [2]. Unfortunately, most of them still are exposed to high-risk injury due to improper workstations and insufficient training in basic biomechanical principles provided to the employees [3].

Upper extremity musculoskeletal disorders (UEMSDs), also known as ULDs have been regularly described as elevated risks for the workers where they are exposed to psychosocial work factors [4]. In fact, ULDs are widely known as the leading cause of work-related disability within various countries ${ }^{1}[5]$. Generally, supermarket cashiers are the common workers having the risk of experiencing musculoskeletal disorders of the upper limb [6], commonly the carpal tunnel syndrome (CTS) [7]. They tend to perform repetitive actions and light manual material handlings involving scanning and dealing with products,

${ }^{1}$ Corresponding author: mnasrull@uthm.edu.my 
where these actions are reported as a risk factors contributed to the upper limb musculoskeletal disorders [8]. These include body posture and movements (sitting, standing, lifting, pulling, and pushing) and environmental factors (noise, lighting, temperature, and humidity) [9]. Besides that, repetition, overload, awkward positions or the combination of any of these are also some factors that contributed to the disease [10]. Other than grocery industries, workers in mould manufacturing industries also are highly exposed to upper limb disorders due to similar risk factors such as repetitive movements, uncomfortable work postures and excessive work without breaks [11]. The same applies for workers dealing with wall plastering job in construction industry [12].

There were various methods used for the assessment of risk factors for the upper limb disorders. The Disabilities of the Arm, Shoulder and Hand (DASH) questionnaire [13] acts as a structured interview in determining the prevalence of ULDs. On the contrary, the Assessment of Repetitive Tasks (ART) [14], Job Strain Index (JSI) [15] and Occupational Repetitive Actions (OCRA) Checklist [16] act as a direct observation in examining the involvement of risk factors. The objective of this research is to review the methods used in assessing risk factors for the upper limb disorders among cashiers in grocery retail industries.

\section{Methods used in assessing risk factors for upper limb disorders among cashiers in grocery retail industries}

In this paper, the questionnaire and tools used in assessing risk factors among cashiers in grocery retail industries were discussed. The usage of these methods is important to assess the risk factors for the upper limb disorders. The tools dealt with the measurement of symptoms, posture observation, and workplace risk assessment. These tools are beneficial in obtaining crucial surveillance data for the management to determine the disorders and reducing risk injury in a workplace. The relevant questionnaire and tools in the context of grocery industries are discussed.

\subsection{The Disabilities of the Arm, Shoulder and Hand (DASH) questionnaire}

The Disabilities of the Arm, Shoulder and Hand (DASH) Questionnaire is an upper extremity specific outcome measurement that has been introduced back in 1996 by the American Academy of Orthopedic Surgeons in collaboration with other certain organisations [13]. Basically, DASH is a self-report questionnaire aimed to measure physical function and symptoms in subjects with any musculoskeletal disorders of the upper limb.

DASH Questionnaire has additional optional modules. These modules were used to measure symptoms and functions among athletes, artists, and workers who require a high level of function. The module inquires about the impact of the arm, shoulder, and hand problems on the ability to perform tasks during work. Another additional optional module was the sports/art module. The module inquires about the impact of the arm, shoulder, and hand problems on activities such as playing musical instruments or doing sports, or both. The questionnaire in the additional optional module is the same as DASH Questionnaire having five different responses. The scale score was calculated ranging from 0 (no difficulty) to 100 (unable).

The DASH was separated into two sections, the disability/symptom questions which consist of 30 items, the optional work module and performance sport/music module, each consists of 4 items. The values from the completed response were summed and averaged to produce a score out of five. These values were converted according to Equation 1, to be 
compared to other measurement scaled on 0 to 100 . The higher the score the greater the disability.

$$
\begin{aligned}
\text { DASH Disability Score }= & {[\text { (Sum of response })-1] /(\mathrm{n}(\text { where } \mathrm{n} \text { is the number of }} \\
& \text { completed response }) \times 25
\end{aligned}
$$

\subsection{Assessment of Repetitive Tasks (ART)}

The Assessment of Repetitive Tasks (ART) was developed by Health and Safety Executive in the United Kingdom back in 2009. ART tool was developed to assess risk on tasks that require repetitive movements of the upper limbs [14].

ART tool was developed based on the Manual Handling Assessment Charts [17] which uses a logical flowchart process and 'traffic light' system to grade the risks. The ART tool was composed of some factors in assessing the repetitive movements of the upper limbs. These factors consist of four stages, namely frequency and repetition of movements (A), force (B), awkward postures (C), and additional factors (D). The duration stated in additional factors represents the duration of the task performed by the worker. The duration of the task was used to determine the duration multiplier.

Table 1. Duration Multiplier of the Duration Task [14]

\begin{tabular}{cc}
\hline Duration Task & Duration Multiplier \\
\hline$\leq 2$ hours & x 0.5 \\
$2 \leq$ hours $\leq 4$ & x 0.75 \\
$4 \leq$ hours $\leq 8$ & x 1 \\
$\geq 8$ hours & x 1.5 \\
\hline
\end{tabular}

In order to calculate the task score, the scores of all factors from Stage A to D was added. The task score was then multiplied by duration multiplier. Finally, the exposure score was determined. Low exposure level should consider each individual circumstance if the score recorded was in the range of 0 to 11 . On the other hand, medium requires further investigation if the score recorded was in the range of 12 to 21. Lastly, further investigation is required for high exposure level if the score recorded was above 22.

$$
\text { Task Score }=\mathrm{A}+\mathrm{B}+\mathrm{C}+\mathrm{D}
$$

Task Score $\mathrm{x}$ Duration Multiplier $=$ Exposure Score

Table 2. Exposure level for ART risk factors [14]

\begin{tabular}{ccc}
\hline Exposure Score & & Exposure Level \\
\hline $0-11$ & Low & Consider individual circumstances \\
$12-21$ & Medium & Further investigation required \\
22 or more & High & Further investigation required urgently \\
\hline
\end{tabular}

\subsection{Job Strain Index (JSI)}

In 1995, the Job Strain Index (JSI) was developed by J. Steven Moore and Arun Garg. This tool was developed to analyse the single task jobs in which there are similar motions or only one repeated motion with the same effort level needed throughout the task [15]. The JSI needs both the qualitative and quantitative measurements of six variables, namely intensity of exertion (IE), duration of exertion per cycle (DE), efforts per minute (EM), hand/wrist posture (HWP), speed of work (SW), and duration of task per day (DD) [18]. Duration of exertion per cycle, efforts per minute and duration of task per day are the three 
quantitative variables while the intensity of exertion, hand/wrist posture and speed of work are categorised as qualitative variables. Each variable has its own rating score ranging from one to five.

Table 3. Rating values for each variable [15]

\begin{tabular}{ccccccc}
\hline $\begin{array}{c}\text { Rating } \\
\text { Values }\end{array}$ & IE & DE & EM & HWP & SW & DD \\
\hline 1 & Light & $<10$ & $<4$ & $\begin{array}{c}\text { Very } \\
\text { good }\end{array}$ & $\begin{array}{c}\text { Very } \\
\text { slow }\end{array}$ & $\leq 1$ hour \\
2 & $\begin{array}{c}\text { Somewhat } \\
\text { hard }\end{array}$ & $\begin{array}{c}10- \\
29\end{array}$ & $4-8$ & Good & Slow & $1-2$ hours \\
3 & Hard & $30-$ & $9-14$ & Fair & Fair & $2-4$ hours \\
4 & Very hard & $50-$ & $15-19$ & Bad & Fast & $4-8$ hours \\
5 & Near maximal & $\geq 80$ & $\geq 20$ & Very bad & Very fast & $\geq 8$ hours \\
\hline
\end{tabular}

In order to calculate the JSI score, the multipliers should be determined first. The multipliers for each variable were based on the rating values.

Table 4. Multipliers for each variable [15]

\begin{tabular}{ccccccc}
\hline Rating Values & IE & DE & EM & HWP & SW & DD \\
\hline 1 & 1 & 0.5 & 0.5 & 1.0 & 1.0 & 0.25 \\
2 & 3 & 1.0 & 1.0 & 1.0 & 1.0 & 0.50 \\
3 & 6 & 1.5 & 1.5 & 1.5 & 1.0 & 0.75 \\
4 & 9 & 2.0 & 2.0 & 2.0 & 1.5 & 1.0 \\
5 & 13 & 3.0 & 3.0 & 3.0 & 2.0 & 1.5 \\
\hline
\end{tabular}

The JSI scores were calculated by multiplying all the variables (intensity of exertion, duration of exertion, hand/wrist posture, and speed of work and duration per day) multipliers as described in Equation 4. The JSI exposure was then determined using the JSI score. The job was considered safe if the JSI score recorded was lower than 3 . If the JSI score recorded was in the range of 3 to 7 , the job may increase the risk for distal upper extremities disorders. Furthermore, if the JSI score recorded was higher than 7, then the job was considered hazardous to the workers.

$$
\text { JSI = IE x DE x EM x HWP x SW x DD }
$$

Table 5. Exposure level for JSI risk factors [15]

\begin{tabular}{cc}
\hline JSI Score & JSI Exposure Level \\
\hline JSI $\leq 3$ & The job is probably safe \\
$3<$ JSI $<7$ & The job may increase the risk for distal upper extremity \\
JSI $\geq 7$ & disorders \\
\hline
\end{tabular}

\subsection{Occupational Repetitive Actions (OCRA) Checklist}

The Occupational Repetitive Actions (OCRA) Checklist was developed by Occhipinti \& Colombini back in 1996. Basically, this method was developed to analyse the workers' 
exposure to various upper limb risk factors such as repetitiveness, force, awkward postures and movements, lack of recovery periods, and other additional features [16].

OCRA Checklist consists five parts where each devoted to the analysis of different risk factor. Originally, there is four main risk factors namely lack of recovery time, movement frequency, force, and awkward postures with stereotyped movements. The other additional risk factors considered were vibration transmitted to the hand-arm system, ambient temperatures below $0^{\circ} \mathrm{C}$, precision work, and the usage of inadequate gloves.

OCRA score can be calculated once the assessment of each part has been completed. OCRA score is the sum of frequency, force, recovery, posture, and additional risk factors, which then multiplied with the multiplier for the net duration of the repetitive task as described in Equation 5. The multiplier was based on the total net duration of the repetitive tasks recorded previously.

Table 6. Multiplier of the net duration repetitive tasks [16]

\begin{tabular}{lll}
\hline \multicolumn{3}{c}{ Duration Multiplier of the Net Duration Repetitive Tasks } \\
\hline $60-120 \min =0.5$ & $241-300 \min =0.85$ & $421-480 \mathrm{~min}=1$ \\
$121-180 \min =0.65$ & $301-360 \min =0.925$ & sup $480=1.5$ \\
$181-240 \mathrm{~min}=0.75$ & $361-420 \min =0.95$ & \\
\hline
\end{tabular}

The final score was then evaluated and the exposure level was determined. The final score below 7.5 was considered as an acceptable risk of exposure level. The final score ranging from 7.6 to 11.0 and 11.1 to 14.0 were considered as very low and light risk of exposure level, respectively. Furthermore, the final score ranging from 14.1 to 22.5 was considered medium exposure level and final score above 22.6 was considered as high-risk exposure level.

$$
\text { OCRA Score }=\left(\begin{array}{c}
\text { Frequency }+ \text { Force }+ \text { Recovery }+ \text { Posture }+ \text { Additional }) \\
\text { x Duration Multiplier }
\end{array}\right.
$$

Table 7. Exposure level for OCRA Checklist risk factors [16]

\begin{tabular}{ccc}
\hline OCRA Checklist Score & \multicolumn{2}{c}{ Exposure Level } \\
\hline Up to A 7.5 & Green & No risk (acceptable) \\
$7.6-11.0$ & Yellow & Very low risk \\
$11.1-14.0$ & Red light & Light risk \\
$14.1-22.5$ & Red medium & Medium risk \\
$\geq 22.6$ & Red high & High risk \\
\hline
\end{tabular}

\section{Conclusion}

To sum up, appropriate tools used are an important part of an ergonomic assessment. This research provides an overview of various methods used for the assessment of risk factors for the upper limb disorders among cashiers in grocery retail industries. These methods are appropriate to be used as they focused on respondents experiencing repetitive movements. Apart from the mentioned tools, there are various tools able to analyse tasks, determining symptoms, examine risk level, design work, and collection of data. There are some significant problems still experienced by the developers and ergonomic users when using these methods and tools, such as in developing methods integrating with other methods, methods related to ergonomic theory, to ease the applications, and also in proving the reliability and validity of the methods used. 
This paper is funded by Office for Research, Innovation, Commercialization and Consultancy Management (ORICC) and Universiti Tun Hussein Onn Malaysia (UTHM) under Geran Penyelidikan Pascasiswazah Scheme (GPPS, Vot U800).

\section{References}

[1] US Department of Labor. (2000). Ergonomics: The Study of Work. OSHA 3125, 2000. Retrieved from www.osha.gov.

[2] OSHA, Occupational Safety and Health Act. (2004). Guidelines for Retail Grocery Stores : Ergonomics for the Prevention of Musculoskeletal Disorders. OSHA, (1), $1-19$.

[3] A. Shinnar, J. Indelicato, M. Altimari, and S. Shinnar. (2004). Survey of ergonomic features of supermarket cash registers. International Journal of Industrial Ergonomics, 34(6), 535-541.

[4] J. K. Park and S. H. Jang. (2010). Association between Upper Extremity Musculoskeletal Disorders and Psychosocial Factors at Work: A Review on the Job DCS Model's Perspective. Safety and Health at Work, 1(1), 37-42.

[5] M. N. A. Rahman and N. S. A. Razak. Review on Pen and Paper Based Observational Methods for Assessing Work-related Upper Limb Disorders. Indian Journal of Science and Technology, 9, 1-11 (Special Issue) (2016).

[6] L. Forcier, C. Lapointe, M. Lortie, P. Buckle, I. Kuorinka, J. Lemaire, et al. (2008). Supermarket workers: their work and their health, particularly their selfreported musculoskeletal problems and compensable injuries. Work (Reading, Mass), 30(4), 493-510.

[7] D. Roman-liu, J. Bugajska, and T. Tokarski. (2014). Comparative Study of Upper Limb Load Assessment and Occurrence of Musculoskeletal Disorders at Repetitive Task Workstations. Industrial Health, 52, 461-470.

[8] K. R. Lehman, J. P. Psihogios, and R. G. Meulenbroek. (2001). Effects of sitting versus standing and scanner type on cashiers. Ergonomics, 44(7), 719-738.

[9] J. Dul, B. Weerdmeester. (2008). Ergonomics for Beginners: A Quick Reference Guide. CRC Press.

[10] M. N. A. Rahman, F. A. Aziz, and R. M. Yusuff. (2010). Survey of body part symptoms among workers in a car tyre service centre. Journal of Human Ergology, 39(1), 53-56.

[11] M. N. A. Rahman, H. R. Haq, M. F. Hassan, M. A. Arifin, M. Z. Yunos, S. A. Bakar. et al. Musculoskeletal discomfort among workers in mould manufacturing industry. ARPJ Journal of Ergineering and Applied Sciences, 10(15), 6269-6273 (2015).

[12] M, N. A. Rahman, M. R. A. Rani and M. J. Rohani. (2012). Investigation of workrelated musculoskeletal disorders in wall plastering jobs within construction industry. WORK: A Journal of Prevention, Assessment and Rehabilitation, 43 (4), 507-514.

[13] P. L. Hudak, P. C. Amadio, and C. Bombardier. (1996). Development of an upper extremity outcome measure: The DASH (disabilities of the arm, shoulder, and head). American Journal of Industrial Medicine, 29(6), 602-608.

[14]HSE. Health \& Safety Executive. (2010). Assessment of Repetitive Tasks of the upper limbs (ART tool), 1-16.

[15] J. S. Moore and A. Garg. (1995) 'The strain index: a proposed method to analyze jobs for risk of distal upper extremity disorders', American Industrial Hygiene Association Journal, 56 (5), 443-458. 
[16]E. Occhipinti and D. Colombini. (1996). Alterazioni muscolo-scheletriche degli arti superiori da sovraccarico biomeccanico: metodi e criteri per l'inquadrament o dell'esposizione lavorativa. La Medicina del Lavoro, 87(6), 491-525.

[17] S. C. Monnington, A. D. Pinder and C. Quarrie. (2002). Development of an inspection tool for manual handling risk assessment. (Sheffield, Health and Safety Laboratory), HSL. Internal Report ERG/02/20.

[18] P. Drinkaus, D. S. Bloswick, R. Sesek, C. Mann, and T. Bernard. (2005). Job level risk assessment using task level strain index scores: a pilot study. International Journal of Occupational Safety and Ergonomics : JOSE, 11(2), 141-152. 